\title{
Test-taking behavior under formula and number-right scoring conditions
}

\author{
BARBARA S. PLAKE and STEVEN L. WISE \\ University of Nebraska-Lincoln, Lincoln, Nebraska \\ and \\ ANNE L. HARVEY
Educational Testing Service, Princeton, New Jersey
}

\begin{abstract}
Plake and Wise (1986) identified several research approaches that have the potential for adding information about guessing behavior on multiple-choice items. These approaches focus on the types and characteristics of items that tend to be omitted under correction-for-guessing instructions, and investigate the item-consideration strategies of examinees. This study employed several of these techniques. Results demonstrate that (1) students do not adjust their item-marking behavior when correction-for-guessing instructions are imposed or removed, and (2) students, in general, use strategies other than elimination of alternatives when determining the correct answer to a multiple-choice item under correction-for-guessing directions.
\end{abstract}

Most studies that have addressed examinees' testtaking behavior on multiple-choice tests have focused on one particular aspect of test-taking behavior: guessing behavior (e.g., Rowley \& Traub, 1977; Slakter, 1968). One important factor that has been shown to affect examinees' guessing behavior is the utilization of specific test instructions and scoring procedures. Correction-for-guessing directions usually instruct examinees to omit an item for which no options can be identified as incorrect. Implicitly, this assumes an option-elimination strategy for item consideration by the examinees. If examinees do not typically adopt an option-elimination strategy when they take an examination that uses formula scoring, the accompanying correction-for-guessing directions may be misleading.

Plake and Wise (1986) identified several research approaches that have the potential to add additional information about guessing behavior of examinees on multiplechoice test items. The purpose of the present study was to operationalize some of the research approaches targeted by Plake and Wise. In particular, the study examined the self-reported test-taking behaviors of examinees by asking examinees direct questions regarding test-taking strategies, procedures used when considering multiple-choice questions, and perceptions about guessing on tests in general.

\section{METHOD}

A 40-item multiple-choice "practice" final examination and a threepart questionnaire regarding test-taking behavior were administered during the last week of class in the Fall 1984 semester to 658 students from

Address communications to Barbara S. Plake, Department of Educational Psychology, 135 Bancroft Hall, University of Nebraska-Lincoln, Lincoln, NE 68588-0348. several undergraduate educational psychology classes at a large midwestern university. The practice examination was to serve as an "orienting' experience preceding self-report of test-taking behavior. The practice examinations consisted of two parts (Parts I and II) and two sets of directions (Correction for Guessing [CFG] and No Correction for Guessing [NCFG]). To alleviate order effects, the order of these parts and directions were counterbalanced to produce four practice test forms: (1) CFG directions, Part I, NCFG directions, Part II; (2) CFG directions, Part II, NCFG directions, Part I; (3) NCFG directions, Part I, CFG directions, Part II; (4) NCFG directions, Part II, CFG directions, Part I. These forms were randomly distributed to the students.

Next, a three-part self-report questionnaire was administered. Part 1 was identical to the questionnaire used by Frary, Cross, and Sewell (1985); it assessed the effects of the varying directions on guessing behavior. Parts 2 and 3 were specifically designed for this study and focused on attitudes about guessing and strategies employed by examinees in considering and selecting answers to multiple-choice items. Part 2 consisted of a 5 -item questionnaire that assessed the procedures used by individual students when considering multiple-choice items under correction-for-guessing directions. Part 3 consisted of 10 Likert-type items that were designed to measure attitudes about guessing. For each of the 10 items, students recorded their degree of agreement using a 1-5 scale $(1=$ strongly disagree to $5=$ strongly agree $)$.

\section{RESULTS}

Part 1 of the questionnaire assessed students' understanding of formula scoring and their test-taking behavior under formula scoring and number-right directions. When asked how they believed their test scores would be computed when formula scoring was imposed, 563 (82\%) correctly stated that their scores would be computed as the number of questions answered correctly minus a fraction of the questions answered incorrectly. Furthermore, 69\% recognized that formula-scoring directions advised them to omit answering a question if they had to guess among all the choices. Moreover, $78 \%$ of the examinees realized that number-right directions advised them to not omit any questions under any circumstances. Therefore, it is 
fairly clear that these examinees not only understood the mathematical adjustment penalty that would occur for incorrect answers under formula-scoring directions, but also could articulate the implications of these directions for omitting items.

However, when these students were asked about their own test-taking behavior, their knowledge about the consequences of guessing under formula scoring was not necessarily followed in their reported test-taking practice. Fifty percent of the students reported that they did not adopt a more conservative guessing strategy for those questions to be formula scored than for those to be scored according to number right. Furthermore, over $40 \%$ of the examinees agreed with the statement that they tend to more or less ignore test directions and "do what comes naturally" when taking a multiple-choice test. Therefore, responses to Part 1 of the questionnaire identified that many students disregarded (or ignored) the directions and elected to randomly guess on multiple-choice examinations.

Part 2 of the questionnaire focused specifically on the strategies examinees reported using when considering multiple-choice items under correction-for-guessing instructions. Fifty-three percent of the students reported that they answered every item, regardless of whether or not they knew the correct answer. When asked what elimination strategy (if any) they used when selecting an alternative under correction-for-guessing directions, $56 \%$ of the students reported that, under these situations, they initially eliminate incorrect choices and then, even if no alternatives were eliminated, guess among the remaining choices. Thirty-five percent of the respondents reported following the more appropriate strategy of only guessing among the remaining alternatives if at least one choice could be eliminated as incorrect (which is the strategy assumed for the correction-for-guessing scoring algorithm).

Next, the students were asked to select how they determine the correct answer on a multiple-choice test with correction-for-guessing directions. Of the four alternatives listed, one modeled the typical "elimination" strategy assumed by the formula-guessing algorithm: "I read the question and then I read the alternatives, looking for alternatives that I know are incorrect. From the remaining group of alternatives, I select the best answer." Only $30 \%$ of the examinees responded that they used this strategy in determining the correct answer. Other frequently selected strategies were, "I read the question and then I read the alternatives to determine which one is the correct answer" (27\%), and "I read the question, and when I have formulated the correct answer in my mind, I read all the alternatives to determine which one is the correct answer' (26\%). Thus, the analysis of Part 2 revealed that the majority of students use an item-consideration strategy that is not consistent with elimination of alternatives. Instead, most students (70\%) reported that they read over and directly select the correct alternative through a logical analysis of the items and alternatives.
Table 1

Factor Pattern Matrix Attitudes About Guessing

\begin{tabular}{|c|c|c|c|}
\hline Item & $F_{1}$ & $F_{2}$ & $F_{3}$ \\
\hline $\begin{array}{l}\text { Guessing increases } \\
\text { score }\end{array}$ & -.18 & .46 & .09 \\
\hline Not right to guess & .55 & .47 & .24 \\
\hline $\begin{array}{l}\text { Correct answer } \\
\text { or omit }\end{array}$ & 36 & -08 & 17 \\
\hline Cheating to guess & .63 & -.05 & -.13 \\
\hline $\begin{array}{l}\text { Randomly guess when } \\
\text { out of time }\end{array}$ & -.01 & -.02 & -.37 \\
\hline Dishonest to guess & .64 & -.09 & -.17 \\
\hline $\begin{array}{l}\text { Guessing okay since } \\
\text { mimics life }\end{array}$ & -.14 & .24 & -.11 \\
\hline $\begin{array}{l}\text { I'm luckier than } \\
\text { others }\end{array}$ & -.04 & .36 & .10 \\
\hline Eliminate alternatives & -.11 & .16 & .34 \\
\hline $\begin{array}{l}\text { Guessing against my } \\
\text { personal convictions }\end{array}$ & .65 & -.16 & -.04 \\
\hline
\end{tabular}

Note $-F_{1}=$ morality. $F_{2}=$ beliefs about guessing. $F_{3}=$ guessing strategies.

Part 3 of the questionnaire was analyzed to identify attitudes about guessing on multiple-choice items. The responses to the 10 items were correlated, and the correlation matrix was submitted to a principal-axis factor analysis, using the eigenvalue greater than one criterion for factor retention. Three factors were identified, accounting for $50 \%$ of the variance. A Varimax rotation of these factors and $\mathrm{a} \lambda>.30$ as the criterion for salient factor loadings yielded a structure interpreted to yield the following three factors: morality, beliefs about guessing, and guessing strategies. The factor pattern matrix is shown in Table 1.

\section{DISCUSSION}

The purpose of this study was to operationalize some research approaches to investigations of guessing behavior that were suggested by Plake and Wise (1986). The results of the study suggest that although students tend to be able to accurately comprehend the implications and penalties of formula scoring on test performance, their personal testtaking behavior under correction-for-guessing directions is less than optimal for maximizing their scores under correction-for-guessing scoring. For example, they related that their guessing behavior on tests is independent of their determining penalty information by reading the directions and, when considering an item under correction-for-guessing instructions, their analysis of the alternatives tends to be based on some logical strategy. Attitudes about guessing on multiple-choice tests, furthermore, is complex, representing three dominant factors: morality, beliefs about guessing, and guessing strategies.

Additional research is needed to clarify and amplify the results of this research project. It would be interesting to consider the guessing beliefs and strategies of selected groups of examinees, for example of males and females, and of ethnic/racial groups. Furthermore, a comparison of test-taking strategies for high and low scorers may illuminate systematic errors in test-taking strategies for low-scoring examinees.

\section{REFERENCES}

Frary, R. B., Cross, L. H., \& Sewell, E. H. (1985, April). Partial information and the "correction" for guessing. Paper presented at the annual meeting of the National Council on Measurement in Education, Chicago, IL. 
Plake, B. S., \& Wise, S. L. (1986). Dynamics of guessing behavior: Between-group versus within-group designs. Bulletin of the Psychonomic Society, 24, 251-253.

Rowley, G. L., \& Traub, R. E. (1977). Formula scoring, numberright scoring and test-taking behavior. Journal of Educational Measurement, 14, 15-22.
Slakter, M. J. (1968). The effect of guessing strategy on objective test scores. Journal of Educational Measurement, 29, 115-128.

(Manuscript received for publication November 10, 1987.) 